\title{
A Comprehensive Mechanotransduction Model for Tactile Feedback based on Multi-Axial Stresses at the Fingertip-Contact Interface*
}

\author{
M. R. Valero, N. Hale, J. Tang, L. Jiang
}

\begin{abstract}
This paper presents a mechanotransduction model designed to convert the multi-axial mechanical loads at the fingertip-contact interface into neural-spike trains, the MultiAxial Stress Mechanotransduction (MASM) model. We believe this is a first attempt towards a comprehensive model, accounting for the conversion of measured multi-axial (pressure and shear) stresses at the fingertip-contact interface into spike trains with modelled slow adapting (SA) and rapidly adapting (RA) afferents type I (SAI, RAI) and II (SAII, RAII) based on the properties of those in human fingertips. To illustrate and assess how the MASM model works, artificial data mimicking typical stress stimuli used to evaluate the response of biological afferents were fed to the model and results examined. Subsequently, the suitability of the MASM model for real tactile applications was preliminary tested by inputting to the model real life, measured pressure and shear data in a fingertip 'press-push-lift' action. The response of the modelled SA and RA afferents was analysed and qualitatively compared to biological data reported in literature. Initial results show that it is possible to codify the mechanical contact tactile information measured by multi-axial sensor systems in a bio-inspired fashion, thus reproducing relevant features similar to those produced by biological mechanoreceptors.
\end{abstract}

\section{INTRODUCTION}

Human hand tactile dexterity $[1,2]$, such as in object manipulation and slipping detection $[1,3]$, relies on the tactile feedback provided by mechanoreceptors. In the glabrous skin at the fingertips, these mainly comprise slowly adapting type I (SAI)-Merkel cells, rapidly adapting type I (RAI)-Meissner corpuscles, rapidly adapting type II (RAII)-Pacinian corpuscles, and slow adapting type II (SAII)-Ruffini corpuscles $[1,2]$. Mechanoreceptors produce trains of action potentials in response to the multi-axial (pressure and shear) stresses applied on the skin, with this feedback tactile information ultimately delivered to the brain.

It is envisaged that the next generation of upper limb prostheses will provide a more natural control through enhanced sensory feedback, in a fashion similar to how mechanoreceptors produce neural-spike trains in response to

*This project is funded by the UK's EPSRC through grant EP/M025977/1 (SENSEBACK Project).

M. R. Valero is with the University of Southampton, Faculty of Engineering and the Environment, Highfield Southampton SO17 1BJ, UK (phone: +44 0238059 2443; e-mail: M.Valero@soton.ac.uk).

$\mathrm{N}$. Hale is with the University of Southampton, Faculty of Engineering and the Environment, Highfield Southampton SO17 1BJ, UK (e-mail: nh7g15@soton.ac.uk).

J. Tang is with the University of Southampton, Faculty of Engineering and the Environment, Highfield Southampton SO17 1BJ, UK (e-mail: jt7g13@soton.ac.uk)

L. Jiang is with the University of Southampton, Faculty of Engineering and the Environment, Highfield Southampton SO17 1BJ, UK (e-mail: L.Jiang@soton.ac.uk). tactile stimuli [4-7]. Reviewing the literature, some works have been reported aiming to convert the tactile information into neural spike-trains [4-7]. Recent works have used the mechanical contact information, more specifically pressure values $[5,7]$, as key inputs to develop their models for tactile transduction, predominantly predicting neural-spike trains of SAI afferents. This has provided a valuable insight on means to translate external mechanical stimuli into spike trains.

However, some limitations exist, with other types of afferents, namely SAII [4-7], and stresses -like shear [5, 7], to be also incorporated to the mechanotransduction models. In fact, studies performed on human subjects $[1,2]$ have demonstrated the ability of SA and RA mechanoreceptors at the fingertips to detect and encode multi-axial mechanical stimuli $[1,2]$, with the brain relying on this pressure and shear information for object manipulation and haptic identification [2]. Thus, the development of comprehensive models, with modelled SAI, RAI, RAII and SAII units accounting for the conversion of pressure and shear stresses, is crucial towards a more in-depth covering and understanding of tactile perception, also in order to ensure a more effective and direct tactile feedback.

This paper presents a bio-inspired Multi-Axial Stress Mechanotransduction (MASM) model with potentials for tactile feedback applications. The model is designed to convert the multi-axial mechanical stresses at the fingertipcontact interface into neural-spike trains by artificial SAI, RAI, RAII and SAII mechanoreceptive units. The latter have been modelled based on the characteristics of those existing in human glabrous skin, such as sensing capabilities and firing response. Such a comprehensive model could be potentially exploited in tactile feedback applications, such as in robotics and upper limb neural-based prostheses by converting multi-axial interface stresses at prosthetic hands into patterns of neural activity effected to the nervous system of the prosthesis user through electrical stimulation.

\section{MASM MODEL}

Fig. 1 shows the conceptual diagram of the MASM model. It is comprised of two main sub-models: the transduction and the neural sub-models.

The transduction sub-models translate the measured multiaxial stresses $\sigma_{i}(\mathrm{i}=1,2,3)$, i.e. shear $\mathrm{X}$, shear $\mathrm{Y}$ and pressure respectively, into transmembrane currents $I_{\alpha}(\alpha=$ SAI, RAI, RAII, SAII), which are fed into the neural sub-models. 


\section{Multi-Axial Stress Mechanotransduction}

(MASM) Model

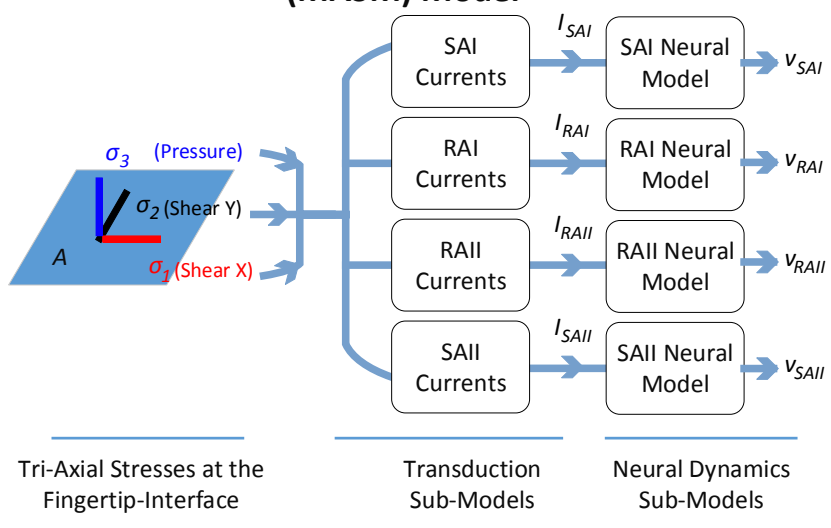

Figure 1. Overview of the Multi-Axial Mechanotransduction (MASM) model. The measured stresses $\sigma_{\mathrm{i}(\mathrm{i}=1,2,3)}$ over the cross-sectional area A are fed to the transduction sub-models for the generation of the transmembrane currents $I_{\alpha(\alpha=\text { SAI, RAI, RAII, SAII })}$ of the artificial SAI, RAI, RAII and SAII afferents in the neural sub-models, which translate the $I_{\alpha}$ currents into the membrane potentials $v_{\alpha(\alpha=\mathrm{SAI}, \mathrm{RAI}, \mathrm{RAII}, \mathrm{SAII})}$ and thus, neural-spike trains.

The neural sub-models contain the neural dynamics models for the generation of the neural spike-trains. They translate the currents $I_{\alpha}(\alpha=$ SAI, RAI, RAII, SAII) from the transduction submodels into the corresponding membrane potentials of the artificial mechanoreceptive units SAI, RAI, RAII and SAII $v_{\alpha}$ ( $\alpha=$ SAI, RAI, RAII, SAII).

Next, the transduction and neural sub-models are explained in more detail.

\section{A. Transduction Sub-Models}

The transduction sub-models translate the measured stresses into currents, analogously to how stresses applied at the SA and RA afferents are transformed into receptors currents across their membranes.

Here, shear X $\left(\sigma_{1}\right)$, shear Y $\left(\sigma_{2}\right)$ and pressure $\left(\sigma_{3}\right)$ stresses are defined as the ratio of the corresponding force $F_{i(\mathrm{i}=1,2,3)}$ to the cross-sectional area $A$ (Fig. 1):

$$
\sigma_{i}=\frac{F_{i}}{A}(i=1,2,3)
$$

Note that $F_{i}(\mathrm{i}=1,2,3)$ correspond to the components of the multi-axial force $F$ applied over $A$, whose magnitude $\|F\|$ can be expressed as (Eq. (1a)):

$$
\|F\|=\sqrt{\sum_{i=1}^{3} F_{i}^{2}}=A \cdot \sqrt{\sum_{i=1}^{3} \sigma_{i}^{2}}
$$

Tactile perception involves the sensing and transduction of assorted tactile information $[1,2]$. Each mechanoreceptor is specialized to convey certain stimuli, with transmembrane currents that can be modelled by $I_{\alpha}(\alpha=$ SAI, RAI, RAII, SAII $)$ functions dependent on specific combinations of $\sigma_{i(\mathrm{i}=1,2,3)}$ and their time derivatives. Namely,

- SAI-Merkel cells are mostly sensitive to static force, responding to indentation and depth [1]. In first order approximation, this can be linked to the magnitude of the normal component of the applied force $F_{3}$ [1], and thus, pressure $\sigma_{3}[5,7]$. From Eq. (1a):

$$
I_{S A I} \approx I_{S A I}\left|\sigma_{3}(t)\right|
$$

- RAI-Meissner corpuscles are mainly insensitive to static force, responding to dynamic skin deformation [1] (velocity). In first order approximation, their response can be modelled as dependent on the first time derivative of the magnitude of the tri-axial applied force $F$. From Eq. (1b), we can write:

$$
I_{R A I} \approx I_{R A I}\left(\frac{\partial}{\partial t}\left|\sqrt{\sum_{i=1}^{3} \sigma_{i}^{2}}\right|\right)
$$

- RAII-Pacinian corpuscles, like RAI afferents are also largely insensitive to static force, and respond to mechanical transients [1] (acceleration). In first order approximation, we can model $I_{R A I I}$ as dependent on the second time derivative of $F$. From Eq. (1b):

$$
I_{R A I I} \approx I_{R A I I}\left(\frac{\partial^{2}}{\partial t^{2}}\left|\sqrt{\sum_{i=1}^{3} \sigma_{i}^{2}}\right|\right)
$$

- SAII-Ruffini corpuscles are sensitive to static force, responding to lateral stretching of the skin [1]. In first order approximation, this can be linked to the magnitude of tangential component of $F$ (i.e., projection over the X, Y plane). From Eq. (1b):

$$
I_{\text {SAII }} \approx I_{\text {SAII }}\left(\left|\sqrt{\sum_{i=1}^{2} \sigma_{i}^{2}}\right|\right)
$$

Reviewing the literature, several versions of linear, power, logarithmic, exponential, hyperbolic log tangent, and non-linear $I$ functions have been proposed [8, 9]. In this paper, linear $I_{\alpha}$ functions have been implemented due to their biological plausibility as well as minimising the complexity of both model and computational engine [5]. In this way, Eqs. (2), (3), (4) and (5) can be rearranged y as:

$$
\begin{gathered}
I_{S A I} \approx k_{S A I} \cdot\left|\sigma_{3}(t)\right|+I_{S A I}^{0} \\
I_{R A I} \approx k_{R A I} \cdot \frac{\partial}{\partial t}\left(\left|\sqrt{\sum_{i=1}^{3} \sigma_{i}^{2}}\right|\right)+I_{R A I}^{0} \\
I_{R A I I} \approx k_{R A I I} \cdot \frac{\partial^{2}}{\partial t^{2}}\left(\left|\sqrt{\sum_{i=1}^{3} \sigma_{i}^{2}}\right|\right)+I_{R A I I}^{0} \\
I_{S A I I} \approx k_{S A I I} \cdot\left|\sqrt{\sum_{i=1}^{3} \sigma_{i}^{2}}\right|+I_{S A I I}^{0}
\end{gathered}
$$


where $\mathrm{k}_{\alpha}\left(\alpha=\right.$ SAI, RAI, RAI, SAII) is the gain and $\mathrm{I}^{0}{ }_{\alpha}$ are offset currents to account for varying thresholds.

\section{B. Neural Sub-Models}

The neural sub-models implement Izhikevich neurons [10] to transform transmembrane currents into spike times.

Izhikevich neurons are an efficient model of the spiking dynamics of neural systems, capable to reproduce spiking and bursting behavior of cortical neurons. They combine the biological plausibility of Hodgkin-Huxley dynamics and the computational efficiency of integrate-and-fire neurons. Dynamics are determined by [11]:

$$
\begin{gathered}
\frac{d v_{\alpha}}{d t}=0.04 v_{\alpha}^{2}+5 v_{\alpha}+140-u_{\alpha}+I_{\alpha} \\
\frac{d u_{\alpha}}{d t}=a_{\alpha} \cdot\left(b_{\alpha} \cdot v_{\alpha}-u_{\alpha}\right)
\end{gathered}
$$

Where $\alpha=$ SAI, RAI, RAI, RAII and with auxiliary afterspike resetting

$$
\text { if } v_{\alpha} \geq 30 \mathrm{mV} \text {, then }\left\{\begin{array}{l}
v_{\alpha} \leftarrow c_{\alpha} \\
u_{\alpha} \leftarrow u_{\alpha}+d_{\alpha}
\end{array}\right.
$$

Here, $v_{\alpha}$ and $u_{\alpha}$ represent the voltage potential and membrane recovery, respectively. By modifying parameters $a_{\alpha}, b_{\alpha}, c_{\alpha}, d_{\alpha}$ it is possible to model tonic and phasic responses, as SA and RA mechanoreceptors, respectively, exhibit [12]. Table I shows the values of the parameters used in this model.

\section{MASM MODEL PERFORMANCE}

To illustrate and assess how the model works, data mimicking typical stress stimuli used to evaluate the response of biological afferents $[1,2]$ were generated here as artificial inputs and fed to the MASM model. The response of the modelled SA and RA afferents was analysed so as to check the capability of the model to quantitatively reproduce relevant features of the corresponding biological units.

Figs. 2(a) and 2(b) show two example stress stimuli for the evaluation of the afferents' response. In each case, three main phases can be distinguished:

- (i) Ramp-up phase (from $\mathrm{t}=0.1 \mathrm{~s}$ to $\mathrm{t}=0.4 \mathrm{~s}$ ). During this phase, the contact between the finger(s) and the probe/external object(s) is established, with stresses steadily increasing to a certain peak value.

- (ii) Plateau phase (from $\mathrm{t}=0.4 \mathrm{~s}$ to $\mathrm{t}=0.9 \mathrm{~s}$ ). In this phase, stresses reach their peak value and stabilise.

- (iii) Ramp-down phase (from $\mathrm{t}=0.9 \mathrm{~s}$ to $\mathrm{t}=1.2 \mathrm{~s}$ ). Contact is released, and stresses decrease until specific offset values.

\begin{tabular}{|c|c|c|c|c|}
\hline \multirow{2}{*}{$\alpha$} & \multicolumn{4}{|c|}{ Izhikevich Neuron Parameters } \\
\cline { 2 - 5 } & $\boldsymbol{a}$ & $\boldsymbol{b}$ & $\boldsymbol{c}$ & $\boldsymbol{d}$ \\
\hline SAI & 0.02 & 0.2 & -65 & 6 \\
\hline RAI & 0.02 & 0.25 & -65 & 6 \\
\hline RAII & 0.02 & 0.25 & -65 & 6 \\
\hline SAII & 0.02 & 0.2 & -65 & 6 \\
\hline
\end{tabular}

As shown, stress profiles in Figs. 2(a) and 2(b) are defined by specific combinations of the rate of change of stress during the ramp-up and ramp-down phase, as well as the magnitude of stress during the plateau phase. The membrane potentials $v_{\alpha}(\alpha=$ SAI, RAI, RAII, SAII) for the stress profiles of Fig. 2(a) and Fig. 2(b), are displayed in Fig. 2(c) and Fig. 2(d), respectively. Fig. 2(e) and Fig. 2(f) show their corresponding peristimulus spike histograms (PSTH).

As it can be seen in Fig. 2(c) and Fig. 2(d), SA afferents adapt slowly $[1,4,5]$, showing an ongoing response related to the strength of maintained stresses $[1,4,5]$, i.e., pressure (SAI) and shear (SAII). This can be more clearly appreciated during the plateau phase. From the comparison between cases $\# 1$ and \#2 in Fig. 2(e) and Fig. 2(f), we can see that SA afferents fire a larger number of spikes as the magnitude of corresponding stresses increase. Thus, SA afferents convey information about the magnitude of the stresses $[1,4,5]$.

Conversely, no spikes are elicited by steady, constant stresses (plateau phase) in RA afferents [1, 4] (Fig. 2(e) and Fig. 2(f)), which mainly respond to stress changes (ramp-up and ramp-down phases) [1, 4]. From Fig. 2(c) and Fig. 2(d), we see that RA afferents show a fast adapting response [1, 4], providing key information about the time at which the mechanical stimulus starts and ends [4]. As shown in Fig. 2(e) and Fig. 2(f), at the onset and the end of the stimulus, the number of spikes elicited from RA afferents is higher than those from their SA counterparts. From Fig. 2(c) and Fig. 2(d), we see that RAI afferents respond during dynamical changes $[1,4]$, i.e., RAI afferents show an ongoing response during all the ramp-up and ramp down phases. On the other hand, RAII are especially responsive to stress transients [1, 4], i.e., transitions between the different phases, as well as at the beginning and end of these.

\section{Test Results And Discussion}

A real life sensor based test was conducted in order to study the potential applicability of the MASM model in real tactile applications.

A sensor system comprising interface sensors of $10 \mathrm{~mm} \mathrm{x}$ $10 \mathrm{~mm}$ in area was used to measure the multi-directional pressure and shear stresses at the fingertip-contact interface. Details of the sensor system and its calibration are described elsewhere [3]. Its decoupled measurement of pressure and shear stresses is particularly advantageous for the evaluation of the proposed model. 


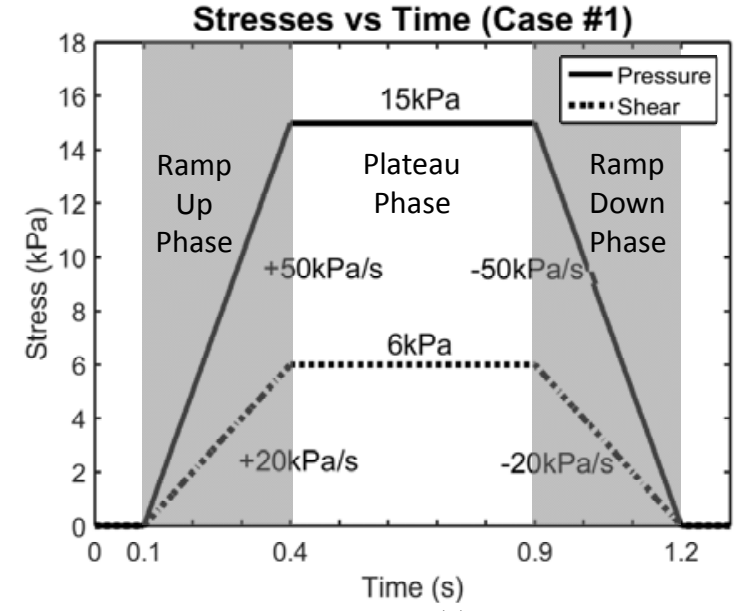

(a)
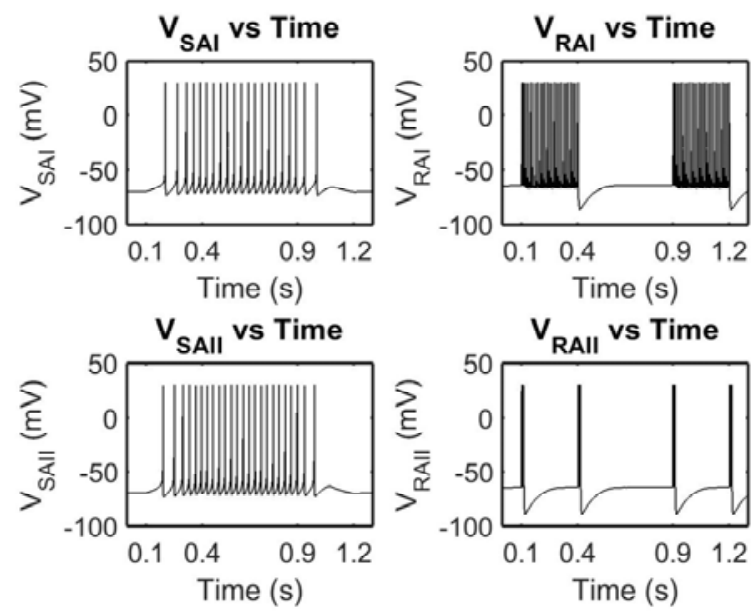

(c)
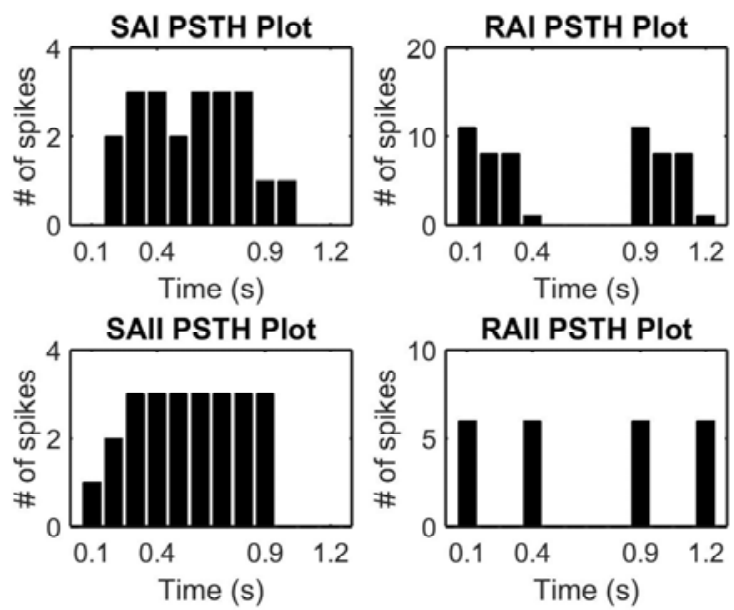

(e)

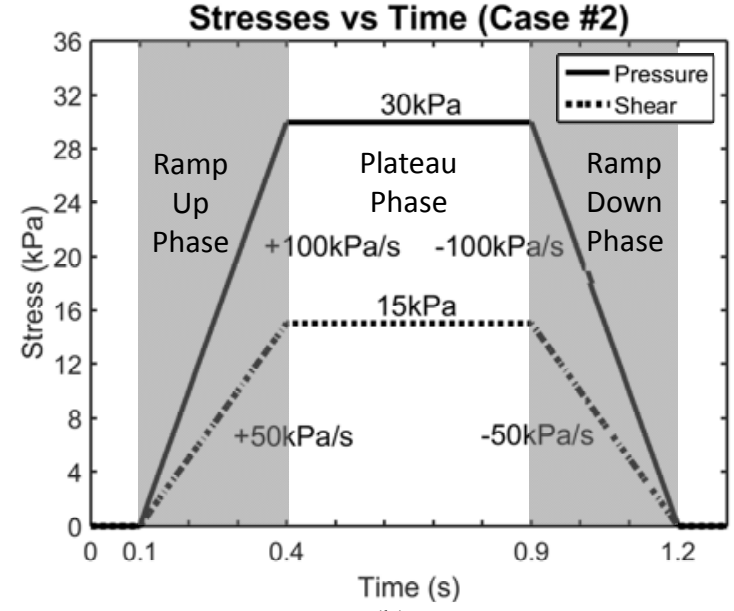

(b)
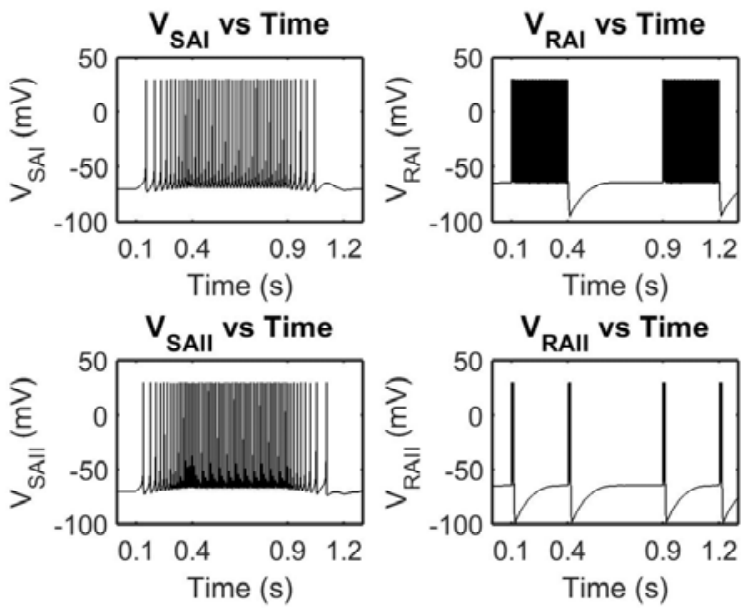

(d)
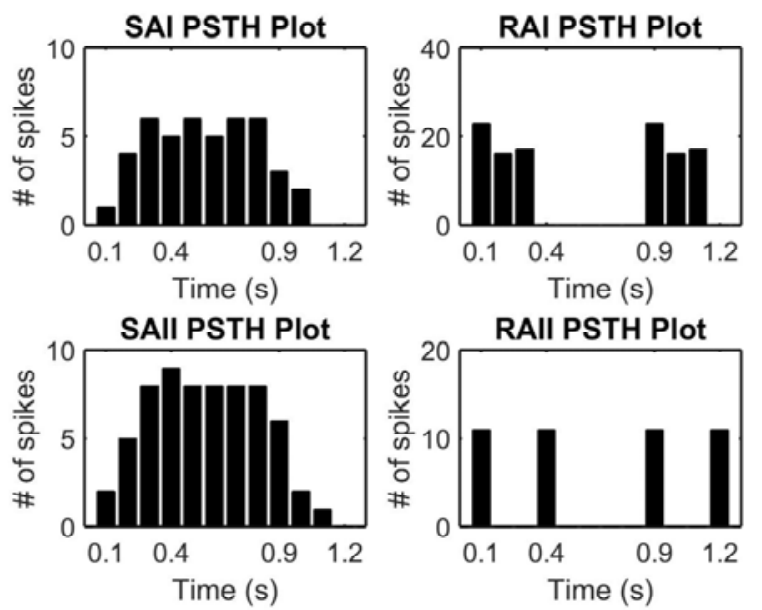

(f)

Figure 2. MASM model's response to example, typical stress stimuli used to evaluate the response of biological afferents. (a) Case \#1 defined by (i) rampup phase: $50 \mathrm{kPa} / \mathrm{s}$ pressure and $20 \mathrm{kPa} / \mathrm{s}$ shear ramp rates (ii) plateau phase: $15 \mathrm{kPa}$ pressure and $6 \mathrm{kPa}$ shear peaks (iii) ramp-down phase: $50 \mathrm{kPa} / \mathrm{s}$ pressure and $20 \mathrm{kPa} / \mathrm{s}$ shear ramp rates, (b) Case \#2 defined by (i) ramp-up phase: $100 \mathrm{kPa} / \mathrm{s}$ pressure and $50 \mathrm{kPa} / \mathrm{s}$ shear ramp rates (ii) plateau phase: $30 \mathrm{kPa}$ pressure and $15 \mathrm{kPa}$ shear peaks (iii) ramp-down phase: $100 \mathrm{kPa} / \mathrm{s}$ pressure and $50 \mathrm{kPa} / \mathrm{s}$ shear ramp rates, (c) Predicted membrane potentials $v$ of the artificial SA and RA afferents for the stress profiles of Fig. 2(a), (d) Predicted membrane potentials of the artificial SA and RA afferents for the stress profiles of Fig. 2(b), (e) Peristimulus spike histogram (PSTH) for Fig. 2(c), (f) PSTH for Fig. 2(d). 
A sensor unit was attached to the index finger of a healthy human subject with no impaired hand function (Fig. 3(a)). The subject was then asked to perform a "press-push-lift" action over a solid surface in the shear Y direction. This action consisted on the following: after establishing contact, the subject is to exert a compressive force (press action) with the index finger of their dominant hand, pushing in direction away from the subject, as shown in Fig. 3(a), and finally releasing the contact by lifting the finger. The subject was asked to perform this task at a comfortable pace as if they were to perform the activity on a daily basis, such as when scrolling over a screen of a smart phone. This study was approved by University of Southampton Ethics and Research Governance Committee (ID: 20847).

The measured pressure and shear stresses are shown in Fig. 3(a), whereas the corresponding sequences of action potentials generated by the artificial SA and RA afferents are shown in Fig. 3(b).

\section{A. Measured multi-axial stresses}

As indicated in Fig. 3(a), the contact was established and the press-push action started approx. $\mathrm{t}=0.1 \mathrm{~s}$, with pressure and shear $Y$ stresses increasing sharply during ramp-up phase (approximately from $\mathrm{t}=0.1 \mathrm{~s}$ to $\mathrm{t}=0.5 \mathrm{~s}$ ) as a result of the press and push action mainly focused along the $\mathrm{Y}$ direction. The smaller magnitude shear $\mathrm{X}$ values point out the existence of a small lateral component of the finger's movement, indicating that the trajectory was not aligned with the shear Y direction.

After pressure, shear $\mathrm{Y}$ and shear $\mathrm{X}$ reaching their peak values in the plateau phase (approximately $\mathrm{t}=0.5 \mathrm{~s}$ to $0.8 \mathrm{~s}$ ). Here, but for a slight decay, stresses remain more stable.

At approximately $\mathrm{t}=0.8 \mathrm{~s}$, pressure, shear $\mathrm{X}$ and shear $\mathrm{Y}$ stresses begin to decrease as contact begins to release until approximately $\mathrm{t}=0.1 \mathrm{~s}$, where the finger lifts and stress values return to baseline values.

\section{B. Neural spike-trains}

As it can be seen in Fig 3(b), at the onset of contact (approximately $\mathrm{t}=0.1 \mathrm{~s}$ ), RA afferents fire the highest number of spikes. In particular, RAI afferents show the highest levels of activity during the ramp-up phase (approximately from $\mathrm{t}=0.1 \mathrm{~s}$ to $\mathrm{t}=0.5 \mathrm{~s}$ ), with increasing pressure and shear stresses as a result of the pressing and pushing actions. This aligns well with biological data, with RAI afferents showing an ongoing response during the ramp-up phase [1,4] and RAII afferents exhibiting a less steady response, mainly firing spikes during pressure and/or shear transients $[1,4]$.

During the plateau phase (approx. from $\mathrm{t}=0.5 \mathrm{~s}$ to $\mathrm{t}=0.8 \mathrm{~s}$ ) the maximum number of spikes are elicited from SA afferents (Fig 3(b)). Compared to RA units, SA afferents adapt slowly $[1,4]$, with more uniform discharge patterns, like commonly observed in biological counterparts [1]. On the other hand, RA afferents reduce significantly their activity, as observed in biological units $[1,4]$.

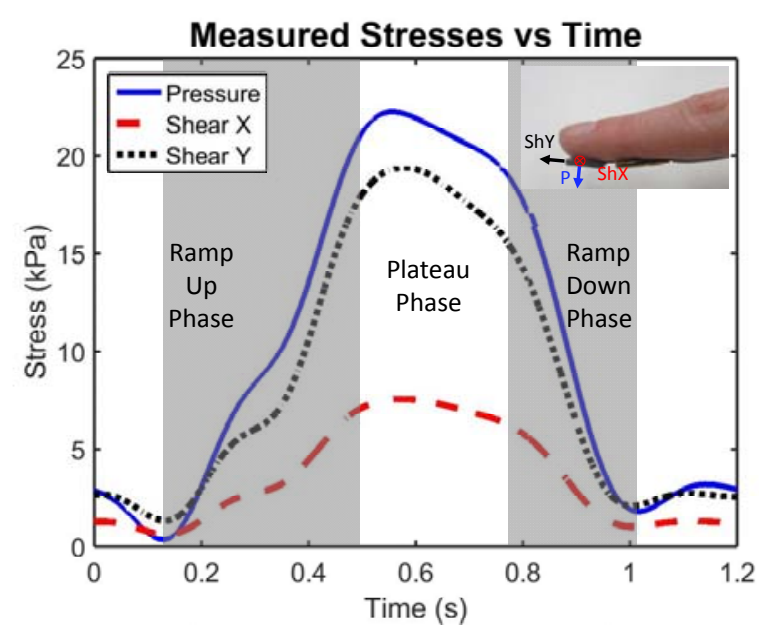

(a)

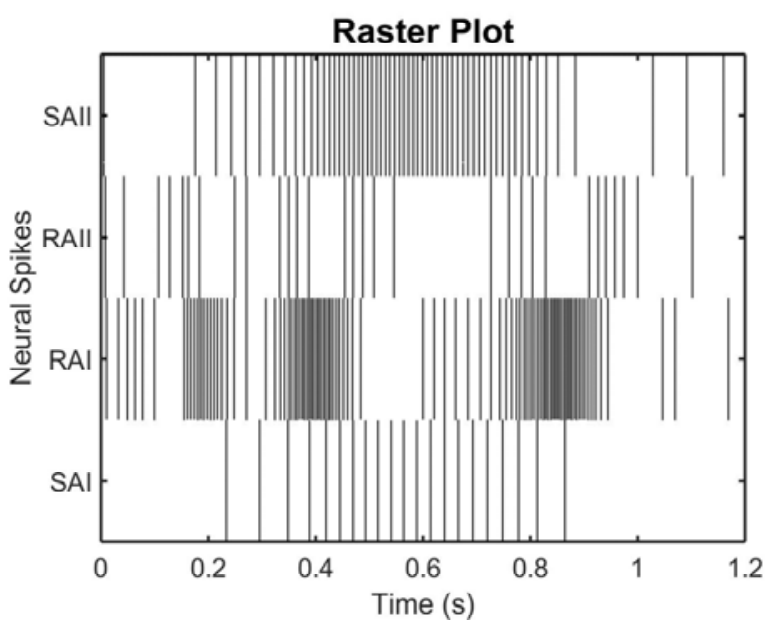

(b)

Figure 3. (a) Measured pressure and shear stresses in a "press-push-lift" activity carried out by a healthy human subject, where three main phases can be distinguished: ramp-up phase, plateau phase, and ramp-down phase

(b) spike times where the membrane potential exceed threshold for the artificial SA and RA afferents.

From Fig. 3(b), we can see that the abovementioned situation is reversed during the ramp-down phase (approximately from $\mathrm{t}=0.8 \mathrm{~s}$ to $\mathrm{t}=1 \mathrm{~s}$ ), with the number of spikes elicited from SA and RA afferents decreasing and increasing, respectively. Like their biological counterparts, RAI afferents show a sustained discharge during most of the ramp-down phase [1, 4], with RAII afferents firing bursts of spikes at the beginning (around $\mathrm{t}=0.8 \mathrm{~s}$ ) and the end of this phase (around $\mathrm{t}=1 \mathrm{~s}$ ) [1,4].

Thus, the qualitative aspects of these results align well with the observed activity of biological afferents $[1,4,5]$. These preliminary results are promising for the further development of the model, namely with fine tuning of the model's parameters and response for specific RA and SA sample populations [4, 5], which could be obtained from biological recorded data. 


\section{CONCLUSION}

A Multi-Axial Stress Mechanotransduction model has been presented in this paper. The MASM model has been designed to convert the mechanical multi-axial (pressure and shear) stresses at the fingertip-contact interface into spike trains with modelled SAI, RAI, RAII and SAII units, based on how mechanoreceptors in human glabrous skin at the fingertips produce trains of action potentials in response to tactile stimuli.

Preliminary results from fingertip data, such as in a "press-push-lift" activity, show that is possible to codify the measured mechanical contact tactile information into neuralspike trains in a bio-inspired fashion, qualitatively reproducing relevant features similar to those produced by biological mechanoreceptors.

Predicted responses align well in qualitative terms with the observed activity of biological afferents, suggesting that the MASM model could be potentially exploited in tactile feedback applications such as in robotics and upper limb neural-based prostheses. Namely, the MASM model could be used to convert multi-axial interface stresses at prosthetic hands into patterns of neural activity, and then effected to the nervous system of the prosthesis user through electrical stimulation. Future works will include further development of the model, with fine tuning of model's response and parameters from biological data.

\section{ACKNOWLEDGMENT}

The authors would like to thank the UK's EPSRC through grant EP/M025977/1 (SENSEBACK Project), Medical Research Council (MRC) and China Scholarship Council (CSC) for support. Supporting data is openly available from the University of Southampton repository at http://dx.doi.org/10.5258/SOTON/xxxxx.

\section{REFERENCES}

[1] A. B. Vallbo, and R. S., Johansson, "Properties of cutaneous mechanoreceptors in the human hand related to touch sensation". Human Neurobiology, Vol. 3, 1984, pp.3-14.

[2] H. E. Wheat, L. M. Salo, A. W. Goodwin, "Human ability to scale and discriminate forces typical of those occurring during grasp and manipulation", The Journal of Neuroscience, Vol. 24, 2004, pp. 3394 -3401 .

[3] M. R. Valero, N. Hale, J. Tang, L. Jiang, M. McGrath, J. Gao, P. Laszczak and D. Moser, "An Interfacial Pressure and Shear Sensor System for Fingertip Contact Applications", Healthcare Technology Letters, Vol. 3, 2016, pp. 280-283.

[4] S. S. Kim, A. P. Sripati, R. J. Vogelstein, "Conveying tactile feedback in sensorized hand neuroprostheses using a biofidelic model of mechanotransduction", IEEE transactions on biomedical circuits and systems, Vol. 3, no. 6, 2009, pp. 398-404.

[5] E. K Kim, S. A Wellnitz, S. M. Bourdon, E. A Lumpkin, G. J Gerling, "Force sensor in simulated skin and neural model mimic tactile SAI afferent spiking response to ramp and hold stimuli", Journal of Neuroengineering and Rehabilitation, Vol. 9, 2012

[6] G. Spigler, C. M. Oddo and M. C. Carrozza, "Soft-neuromorphic artificial touch for applications in neuro-robotics", $20124^{\text {th }}$ IEEE RAS \& EMBS International Conference on Biomedical Robotics and Biomechatronics (BioRob), 2012.

[7] B. C. K. Tee, A. Chortos, A. Berndt, A. K. Nguyen, A. Tom, A. McGuire, Z. C. Lin, K. Tien, W.G. Bae, H. Wang, and P. Mei, "A skin-inspired organic digital mechanoreceptor", Science, Vol. 350, 2015, pp.313-316
[8] J. Hu, Q. Zhao, R. Jiang, R. Wang and X. Ding, "Responses of cutaneous mechanoreceptors within fingerpad to stimulus information for tactile softness sensation of materials", Cognitive Neurodynamics, Vol. 7, 2013, pp.441-447.

[9] Johnsen, S.E. and Trulsson, M., Encoding of amplitude and rate of tooth loads by human periodontal afferents from premolar and molar teeth. Journal of neurophysiology, 93(4), pp.1889-1897, 2005.

[10] E. M. Izhikevich, "Simple model of spiking neurons", IEEE Transactions on Neural Networks, Vol. 14, 2003, pp. 1569-1572.

[11] E. M. Izhikevich, "Which model to use for cortical spiking neurons?", IEEE Transactions on Neural Networks, Vol. 15, 2004, pp. 10631070.

[12] D. Purves, G. J. Augustine, D. Fitzpatrick et al., editors, Neuroscience, $2^{\text {nd }}$ edition. Sunderland (MA): Sinauer Associates; 2001 . 